PROCEEDINGS OF THE

AMERICAN MATHEMATICAL SOCIETY

Volume 129, Number 8, Pages 2385-2393

S 0002-9939(01)05720-3

Article electronically published on January 17, 2001

\title{
SUFFICIENT CONDITIONS FOR A LINEAR FUNCTIONAL TO BE MULTIPLICATIVE
}

\author{
K. SEDDIGHI AND M. H. SHIRDARREH HAGHIGHI
}

(Communicated by David R. Larson)

\begin{abstract}
A commutative Banach algebra $\mathcal{A}$ is said to have the $P(k, n)$ property if the following holds: Let $M$ be a closed subspace of finite codimension $n$ such that, for every $x \in M$, the Gelfand transform $\hat{x}$ has at least $k$ distinct zeros in $\Delta(\mathcal{A})$, the maximal ideal space of $\mathcal{A}$. Then there exists a subset $Z$ of $\Delta(\mathcal{A})$ of cardinality $k$ such that $\hat{M}$ vanishes on $Z$, the set of common zeros of $M$. In this paper we show that if $X \subset \mathbf{C}$ is compact and nowhere dense, then $R(X)$, the uniform closure of the space of rational functions with poles off $X$, has the $P(k, n)$ property for all $k, n \in \mathbf{N}$. We also investigate the $P(k, n)$ property for the algebra of real continuous functions on a compact Hausdorff space.
\end{abstract}

\section{INTRODUCTION}

The well known Gleason-Kahane-Zelazko theorem ([6, 10] states that if $\mathcal{A}$ is any commutative unital Banach algebra and $M$ is a closed subspace with $\operatorname{codim}(M)=1$ such that $M$ does not contain invertible elements, then $M$ is a maximal ideal in $\mathcal{A}$. In other words if $\varphi \in \mathcal{A}^{*}$ is such that $\varphi(a) \in \sigma(a)$ for every $a \in \mathcal{A}$, then $\varphi$ is multiplicative. This theorem has been generalized to higher codimensions (see 1, 12, 13, 7]). To state these generalizations we first need a definition.

Let $\mathcal{A}$ be a commutative complex Banach algebra with identity. We say that $\mathcal{A}$ satisfies the $P(k, n)$ property if the following holds: Let $M$ be a closed subspace of $\mathcal{A}$ of finite codimension $n$. Suppose that for every $x \in M$, the Gelfand transform $\hat{x}$ has at least $k$ distinct zeros in $\Delta(\mathcal{A})$, the maximal ideal space of $\mathcal{A}$. Then there exists a subset $Z$ of $\Delta(\mathcal{A})$ of cardinality $k$ such that $\hat{x}$ vanishes on $Z$ for every $x \in M$. We call $Z$ a set of common zeros of $M$. In fact the hypothesis states that if every $x \in M$ is contained in $k$ maximal ideals $I_{1}^{x}, I_{2}^{x}, \cdots, I_{k}^{x}$ (depending on $x$ ), then there exist $k$ maximal ideals $I_{1}, \cdots, I_{k}$ such that every $x \in M$ is contained in $I_{1}, I_{2}, \cdots, I_{k}$.

Note that if for $k>1$, the $P(k, n)$ holds for $C(X)$ the space of continuous complex valued functions on a compact Hausdorff space $X$, then every point of $X$ is a $G_{\delta}$ set. We now discuss some background history of the subject.

Received by the editors January 31, 1999 and, in revised form, December 17, 1999.

2000 Mathematics Subject Classification. Primary 46J20; Secondary 46J10.

Key words and phrases. Multiplicative linear functional, the $P(k, n)$ property, Banach algebra, maximal ideal.

This research was partially supported by a grant from IPM, The Institute for Studies in Theoretical Physics and Mathematics. 
In [12] Warner and Whitely conjectured that if $X$ is a compact Hausdorff space such that each point of $X$ is a $G_{\delta}$ set, then $C(X)$ satisfies the $P(k, n)$ property. This conjecture was settled by K. Jarosz [7]. Garimella and Rao [5] showed that $C^{n}[a, b]$ and $L^{1}(\mathbf{R})$ satisfy $P(k, n)$. Chen and Cohen [2] have proved that a selfadjoint regular $n$-point spectral Banach algebra satisfies $P(k, n)$. Rao [1] removed the spectrality condition in the Chen and Cohen theorem, thus making the result applicable to $C^{n}[a, b]$. He generalized the above result to any selfadjoint, regular commutative Banach algebra with identity. We would also like to investigate the $P(k, n)$ property. Extensions of the Gleason-Kahane-Zelazko theorem in other directions can be found in [9].

In $\S 2$ we generalize some results in 7$]$ and [5] and show that if $X$ is a compact and nowhere dense subset of the plane, then $R(X)$, the uniform closure of the algebra of rational functions with poles off $X$, has the $P(k, n)$ property for all $k, n \in \mathbf{N}$.

Note that the GKZ theorem does not hold for real Banach algebras. For example let $\mathcal{A}=\operatorname{Re} C[0,1]$ and $\varphi(f)=\frac{1}{2}(f(0)+f(1))$ for all $f$ in $\mathcal{A}$. By the intermediate value theorem $\varphi(f) \in \sigma(f)$ for all $f \in \mathcal{A}$ but $\varphi$ is not multiplicative. This example, in turn, shows that the proof of the GKZ theorem depends heavily on complex analysis techniques. But the GKZ theorem is true for $\operatorname{Re} C(X), X$ compact Hausdorff, if and only if $X$ is assumed to be totally disconnected (see Farnum and Whitely [4]). We may use the definition of the $P(k, n)$ property without any difficulty for real Banach algebras and in $\S 3$ we show that if $X$ is a compact Hausdorff and totally disconnected space, then $\operatorname{Re} C(X)$ has the $P(1, n)$ property for all $n>1$ and also has the $P(k, n)$ property for all $k, n \in \mathbf{N}$, if we further assume that the points of $X$ are $G_{\delta}$. We also show that the $P(1, n)$ property holds for all unital commutative real Banach algebras with a totally disconnected maximal ideal space.

\section{Algebras of Rational functions}

To prove the $P(1, n)$ property for $C(X)$ where $X$ is a compact Hausdorff space, Jarosz proves a lemma [7, Lemma 1] stating that if $X$ is a compact subset of the real line and $p_{1}, \cdots, p_{n}$ are polynomials such that each linear combination of them has a zero in $X$, then there is a common zero for $p_{1}, \cdots, p_{n}$ in $X$. Garimella and Rao [5] generalized this result for those closed subsets $X$ of $\mathbf{C}$ that have an empty interior and called this result the polynomial lemma. The assumption that $X$ has an empty interior is essential as the following example shows:

Let $p_{1}=(i z+1)^{2}$ and $p_{2}=(z+i)^{2}$ on $\overline{\mathbf{D}}$ the closed unit disk. We show that every linear combination of $p_{1}$ and $p_{2}$ has a zero in the disk. To see this note that the Möbius transformation $\frac{i z+1}{z+i}$ sends $\overline{\mathbf{D}} \backslash\{-i\}$ to the lower closed half plane and so the function $h=\frac{(i z+1)^{2}}{(z+i)^{2}}$ sends it to the whole plane. Consequently, every linear combination of $p_{1}$ and $p_{2}$ has a zero in the disk while there is no common zero for $p_{1}$ and $p_{2}$ in the disk.

This example also shows that the assumption of being finite codimensional is essential in the generalizations of GKZ. See also Jarosz [7, §2].

In the proof of Jarosz and Garimella and Rao for the polynomial lemma, the method of several variables is used. Here we prove a generalization of this lemma that uses only elementary complex analysis and needs no assumption of closedness. We only assume that the closure of $X$ in $\mathbf{C}$ has an empty interior, i.e. $X$ is nowhere dense in $\mathbf{C}$. But first we need a lemma. 
Lemma 2.1. Let $X$ be a nowhere dense subset of $\mathbf{C}$ and $f$ be a holomorphic function on a neighborhood of $X$. Then $f(X)$ has an empty interior in $\mathbf{C}$. Consequently, there are infinitely many complex numbers outside $f(X)$.

Proof. Denote by $\bar{X}$ the closure of $X$ in C. By definition, for each $x \in X$ there is a neighborhood of $x$ such that $f$ is holomorphic there. In this proof we restrict ourselves to those neighborhoods $V$ such that $f$ is holomorphic on a neighborhood of $\bar{V}$. Note that $f$ need not be defined on $\bar{X}$.

Let $x$ be in $X$. Two cases are possible:

(i) $f^{\prime}(x) \neq 0$. In this case there exists a bounded open set $V$ containing $x$ such that $f$ is one-to-one on $\bar{V}$. Thus $\bar{V}$ is compact and $f_{1}=\left.f\right|_{\bar{V}}$ is invertible and if $f_{1}(\bar{X} \cap \bar{V})=U$ or equivalently $f_{1}^{-1}(U)=\bar{V} \cap \bar{X}$, then $U$ has an empty interior in $\mathbf{C}$ since otherwise $f_{1}^{-1}$, being holomorphic and nonconstant on $U$, sends open sets to open sets, and so $\bar{V} \cap \bar{X}$ and hence $\bar{X}$ would contain an open set, which contradicts the hypothesis. Therefore $f(\bar{V} \cap \bar{X})=f_{1}(\bar{V} \cap \bar{X})$ has an empty interior in $\mathbf{C}$.

(ii) $f^{\prime}(x)=0$. In this case again two cases may occur. First, there exists a neighborhood $V$ of $x$ such that $f^{\prime}=0$ on $\bar{V}$. Then $f$ is constant on $V$ and hence $f(\bar{V} \cap \bar{X})$ is a singleton and so is closed and has an empty interior. The second possibility is that there exists a neighborhood $V$ such that $f^{\prime}$ has a single zero $x$ in $V$. In this case we may suppose that $V$ is an open ball with center $x$. Choose closed annuli $V_{n}$ such that $\bar{V} \backslash\{x\}=\bigcup_{n=1}^{\infty} V_{n}$. By $(i)$, for all $n=1,2,3, \cdots, f\left(V_{n} \cap \bar{X}\right)$ has an empty interior in $\mathbf{C}$ and therefore $f(\bar{V} \cap \bar{X})=\bigcup_{n=1}^{\infty} f\left(V_{n} \cap \bar{X}\right) \cup\{f(x)\}$ has an empty interior in $\mathbf{C}$ by the Baire category theorem.

Therefore for each $x \in X$, there exists a suitable neighborhood $V$ of $x$ such that $f(\bar{V} \cap \bar{X})$ has an empty interior in C. But $X$ can be covered with a countable union of such suitable neighborhoods $V$. Since for such a neighborhood $V, f(\bar{V} \cap$ $\bar{X})$ is closed and has an empty interior in $\mathbf{C}$, their countable union also has an empty interior by the Baire category theorem. Hence, $f(X)$, being a subset of this countable union, has an empty interior in $\mathbf{C}$.

We also need the following well-known facts from linear algebra.

Lemma 2.2. (i) If a finite dimensional complex or real linear space is a countable union of subspaces, then one of these subspaces must contain the others.

(ii) If a complex or real linear space is a finite union of subspaces, then one of these subspaces must contain the others.

Theorem 2.3. Suppose $X$ is a nowhere dense subset of the plane and $h_{0}, \cdots, h_{n}$ are functions analytic on a neighborhood of $X$ such that each linear combination of them has a zero in $X$. If one of these $h_{j}, 0 \leq j \leq n$, has countably many zeros in $X$, then there exists a common zero for $h_{0}, \cdots, h_{n}$ in $X$.

Proof. Suppose $h_{0}$ has countably many zeros in $X$. Set $Z=\left\{x \in X: h_{0}(x)=\right.$ $0\}$. For a linear combination $h$ of $h_{0}, \cdots, h_{n}$ consider the function $f=h / h_{0}$ on $X^{\prime}=X \backslash Z$. Since $X^{\prime}$ is also nowhere dense in $\mathbf{C}$, by Lemma 2.1 there exists a complex number $\beta \neq 0$ such that $f(x) \neq \beta$ for all $x \in X^{\prime}$. But the equation $h(x)=\beta h_{0}(x)$ has a solution in $X$ and this solution is not in $X^{\prime}$, so that it is in $Z$. Let $M$ denote the complex linear space of linear combinations of $h_{0}, \cdots, h_{n}$, i.e. $M=\left[h_{0}, \cdots, h_{n}\right]$. We have shown that every element in $M$ has a zero in $Z$. For a point $z \in Z$ let $M_{z}=\{f \in M: f(z)=0\}$, so that $M=\bigcup_{z \in Z} M_{z}$. But $Z$ is a 
countable set and by Lemma $2.2(i)$, one of these $M_{z}$ must contain the others, i.e. $h_{0}, \cdots, h_{n}$ has a common zero in $X$.

Corollary 2.4. Under the same hypothesis, if each linear combination of $h_{0}, \cdots, h_{n}$ has at least $k$ zeros in $X$, then there exist $k$ common zeros for $h_{0}, \cdots, h_{n}$ in $X$.

Proof. There exists a common zero for $h_{0}, \cdots, h_{n}$. Remove it from $X$ and continue if necessary.

Corollary 2.5. Suppose $A$ is a linear space of functions on a nowhere dense subset $X$ of the plane each of which is analytic on a neighborhood of $X$, and has at least $k$ zeros in $X$. Furthermore assume that one element of $A$ has finitely many zeros in $X$. Then there exist $k$ common zeros for $A$ in $X$.

For example, $A$ can be a space of polynomials or rational functions.

Proof. Let $p$ in $A$ have only finitely many zeros in $X$. The set $Z=\{x \in X: p(x)=$ $0\}$ is finite and has cardinality $\geq k$. For an arbitrary element $q \in A$, let $h=q / p$, so by Lemma $2.1, h(X \backslash Z)$ has empty interior in $\mathbf{C}$. Thus there exists $\beta \neq 0$ such that $h(x) \neq \beta$ for all $x \in X \backslash Z$. But by the hypothesis, $q-\beta p$ is in $A$ and has $k$ zeros in $X$ and none of the zeros are in $X \backslash Z$; hence all are in $Z$. Therefore $q$ has at least $k$ zeros in $Z$. For a subset $K$ of $Z$ of cardinality $k$, set

$$
A_{K}=\{f \in A: f(x)=0 \text { for all } x \in K\} .
$$

Thus $A=\bigcup_{K} A_{K}$ where $K$ runs over all subsets of $Z$ of cardinality $k$. But $Z$ is a finite set and has only finitely many subsets of cardinality $k$. By Lemma $2.2(i i)$ one of these $A_{K}$ must contain the others, i.e. $A$ has $k$ common zeros in $X$.

Now the $P(k, n)$ property for the algebra of rational functions over a compact nowhere dense subset of the plane follows.

Theorem 2.6. Let $R(X)$ be the uniform closure of the algebra of rational functions $\operatorname{Rat}(X)$ with poles off a compact nowhere dense subset $X$ of the plane. If $M$ is a finite codimensional subspace of $R(X)$ such that every element of it has at least $k$ zeros in $X$, then there are $k$ common zeros for $M$ in $X$. That is, the $P(k, n)$ property holds for $R(X)$.

Proof. Note that the maximal ideal space of $R(X)$ is $X$. Let $M$ be a finite codimensional subspace of $R(X)$ such that each element of $M$ has at least $k$ zeros in $X$. Let $M^{\prime}=M \cap \operatorname{Rat}(X)$. Since $M$ is finite codimensional in $R(X), M^{\prime}$ would be dense in $M$. Also each element of $M^{\prime}$ has at least $k$ zeros in $X$ and hence by Corollary $2.5, M^{\prime}$ has $k$ common zeros in $X$. Now $M$, being the uniform closure of $M^{\prime}$, has $k$ common zeros in $X$.

It follows that the $P(k, n)$ property holds for $C(X)$ whenever $X \subset \mathbf{C}$ is compact and nowhere dense with a connected complement (especially whenever $X \subset \mathbf{R}$ ) since rational functions are dense in $C(X)$ in this case.

The above proof is also applicable to $C^{n}[a, b]$ and to $C^{\infty}[a, b]$, although the latter has no Banach algebra norm, since polynomials are dense in these algebras. 


\section{What happens for $\operatorname{Re} C(X)$ ?}

As stated in the introduction, the GKZ theorem does not hold in general for real Banach algebras and it is true for $\operatorname{Re} C(X), X$ compact, if and only if $X$ is totally disconnected [4]. In fact, if $U$ is a nontrivial connected component of $X$, then we have an abundance of examples of continuous linear functionals $\varphi$ with $\varphi(f) \in \operatorname{Im}(\mathrm{f})$ for all $f$ in $\operatorname{Re} C(X)$ such that $\varphi$ is not multiplicative.

Let $x$ and $y$ be two distinct points of $U$ and $\varphi(f)=\frac{f(x)+f(y)}{2}$. Then for all $f$ in $\operatorname{Re} C(X), \varphi(f) \in \operatorname{Im}(\mathrm{f})$ by the intermediate value theorem and obviously $\varphi$ is not multiplicative. If we consider $U$ as a subset of the dual space of $\operatorname{Re} C(X)$ (the points of $U$ will then be the point mass measures at points of $U$ ), a deeper observation reveals that each element $\varphi$ of $\overline{\mathrm{co}}(U)$, the closure of the convex hull of $U$ in the dual space of $\operatorname{Re} C(X)$, has the property $\varphi(f) \in \operatorname{Im}(\mathrm{f})$ for all $f$ in $\operatorname{Re} C(X)$.

Now we show that if $X$ is a totally disconnected compact Hausdorff space, then $P(1, n)$ is true for $\operatorname{Re} C(X)$ for all $n \in \mathbf{N}$.

Theorem 3.1. If $X$ is a totally disconnected compact Hausdorff space, then $\operatorname{Re} C(X)$ has the $P(1, n)$ property for all $n \in \mathbf{N}$.

Proof. Let $M$ be a finite codimensional subspace of $\operatorname{Re} C(X)$ consisting only of non-invertible elements. Since $X$ is totally disconnected, $X$ can be considered as a closed subspace of a discrete product $\{0,1\}^{J}$ where $J$ is some index set. For a finite subset $J_{0}$ of $J$ denote

$$
M\left(J_{0}\right)=\left\{f \in M: f \text { depends only on the coordinates from } J_{0}\right\}
$$

and

$$
X\left(J_{0}\right)=\left\{x \in X: \quad f(x)=0 \text { for all } f \in M\left(J_{0}\right)\right\} .
$$

Clearly $M\left(J_{0}\right)$ can be considered as a subspace of $\mathbf{R}^{2^{J_{0}}}$ and since each element of $M\left(J_{0}\right)$ is non-invertible; then $X\left(J_{0}\right)$ would be closed (and hence compact) and nonvoid by Lemma $2.2(i)$.

Now for a family $J_{1}, \cdots, J_{m}$ of finite subsets of $J$ let $J_{0}=\bigcup_{i=1}^{m} J_{i}$. Thus $X\left(J_{0}\right) \subseteq \bigcap_{i=1}^{m} X\left(J_{i}\right)$ and since $X\left(J_{0}\right)$ is not empty, then it follows that the class

$$
\left\{X\left(J_{0}\right): J_{0} \text { is a finite subset of } J\right\}
$$

has the finite intersection property and therefore has a nonempty intersection $Z$ which is compact and is a zero set for the subspace

$$
M^{\prime}=\{f \in M: f \text { depends only on a finite number of coordinates from } J\} .
$$

But the linear subspace

$$
Y=\{f \in \operatorname{Re} C(X): f \text { depends only on a finite number of coordinates from } J\}
$$

is a dense subspace of $\operatorname{Re} C(X)$ and $M$ is finite codimensional in $\operatorname{Re} C(X)$, so that $M^{\prime}=Y \cap M$ is dense in $M$ and therefore each element of $M$ is zero on $Z$; that is, $M$ has at least a common zero in $X$.

Now we are ready to prove the $P(k, n)$ property for $\operatorname{Re} C(X)$ if we further assume that the points of $X$ are $G_{\delta}$. In general, all the points of the space $\{0,1\}^{J}$ are not $G_{\delta}$. For example, if $J=[0,1]$, then the point $\overline{\mathbf{0}}$ in $\{0,1\}^{J}$, which is zero in all of its coordinates, is not a $G_{\delta}$ set. 
Theorem 3.2. If $X$ is a totally disconnected compact Hausdorff space such that each point of $X$ is a $G_{\delta}$, then the $P(k, n)$ property holds for $\operatorname{Re} C(X)$ for all $k, n \in$ N.

Proof. Let $M$ be a subspace of $\operatorname{Re} C(X)$ of codimension $n$ such that each element of $M$ has at least $k$ zeros in $X$. By Theorem 3.1, there is at least one common zero for $M$ in $X$. Let $Z=\left\{x_{1}, \cdots, x_{m}\right\}$ denote the set of common zeros for $M$ in $X$. Since the multiplicative functionals on a commutative Banach algebra are linearly independent, it follows that $m \leq n$. If $k \leq m$ we are done. If $k>m$, put

$$
M^{\perp}=\left[\delta_{1}, \cdots, \delta_{m}, \mu_{1}, \cdots, \mu_{m^{\prime}}\right]
$$

where $m+m^{\prime}=n$ and $\delta_{j}$ denotes the point mass measure at $x_{j}$ for $1 \leq j \leq m$. Let

$$
M^{\prime}=\left\{f \in \operatorname{Re} C(X): \mu_{j}(f)=\int f d \mu_{j}=0, \text { for all } j, 1 \leq j \leq m^{\prime}\right\} .
$$

We may suppose, by adding suitable linear combination of $\delta_{1}, \cdots, \delta_{m}$ to each $\mu_{j}$, that $\left|\mu_{j}\right|(Z)=0$ for $1 \leq j \leq m^{\prime}$.

Since there is no common zero for $M^{\prime}$, by Theorem 3.1 there is a function $f \in M^{\prime}$ such that $f \neq 0$ everywhere on $X$. By using the duality notions in the subalgebra

$$
\{f \in \operatorname{Re} C(X): \quad f(Z)=0\},
$$

of $\operatorname{Re} C(X)$, we may choose $f_{j}$ such that $f_{j}$ is zero on $Z$ and $\int f_{i} d \mu_{j}=\delta_{i j}, 1 \leq$ $i, j \leq m^{\prime}$. Let $\epsilon>0$ be such that $g=|f|-\epsilon \sum_{j}\left|f_{j}\right|$ is a strictly positive function. Set $s=\sup _{x \in X} g(x)$. Since each $\mu_{j}$ is regular and zero on $Z$, there exists an open neighborhood $V$ of $Z$ such that $\left|\mu_{j}\right|(V)<\epsilon / s$.

Since $Z$ is a $G_{\delta}$ set, we may choose $h$ in $\operatorname{Re} C(X)$ such that $0 \leq h \leq 1$ on $X, h$ is 1 only on $Z$ and is zero outside $V$. Let $u=g h(\operatorname{sgn} f)$ (since $f \neq 0$ everywhere on $X, \operatorname{sgn} f$ is continuous). Then

$$
\left|\int u d \mu_{j}\right| \leq\left|\mu_{j}\right|(V) \sup _{x \in X} g(x)<\left(\frac{\epsilon}{s}\right) s=\epsilon .
$$

If we consider the function $w=f-u+\sum_{j} \mu_{j}(u) f_{j}$, then a simple computation shows that $w$ is zero on $Z$ and $\int w d \mu_{j}=0,1 \leq j \leq m^{\prime}$. Thus $w$ belongs to $M$. Furthermore, $|w| \geq|f|-|u|-\epsilon \sum_{j}\left|f_{j}\right|=(1-\bar{h}) g>0$, outside $Z$. Hence $w \in M$ has exactly $m$ zeros which is absurd.

Corollary 3.3. If $X$ is a totally disconnected compact Hausdorff space with points $G_{\delta}$ and $M$ is a subspace of codimension $n$ in $\operatorname{Re} C(X)$ such that each element of $M$ has at least $n$ zeros in $X$, then $M$ is an ideal.

In [11, Theorem (0.4)], Rao proved that a complex unital commutative Banach algebra $\mathcal{A}$ has the $P(k, n)$ property if and only if its corresponding semisimple algebra $\frac{\mathcal{A}}{\mathcal{B}}$ has this property where $\mathcal{B}$ denotes the Jacobson radical of $\mathcal{A}$. The proof there is also applicable to real Banach algebras as well. Therefore we conclude the following:

Theorem 3.4. Let $\mathcal{A}$ be a unital real commutative Banach algebra with a totally disconnected maximal ideal space $X$. If $M$ is a finite codimensional subspace of $\mathcal{A}$ such that the Gelfand transform of each element of $M$ has a zero in $X$, then $M$ is contained in a maximal ideal. 
Proof. We can assume that $\mathcal{A}$ is semisimple. Let $\Lambda$ be the Gelfand map from $\mathcal{A}$ into $\operatorname{Re} C(X)$. Then $N=\overline{\Lambda(M)}$ is finite codimensional in $\operatorname{Re} C(X)$ and each element of $N$ is noninvertible in $\operatorname{Re} C(X)$. So by Theorem 3.1 there exists a common zero for $N$ in $X$ and this is a common zero for $M$. Consequently $M$ is contained in a maximal ideal.

\section{Some FURTHER RESUltS}

In 1991, K. Jarosz [9] posed the following problems:

Problem 1. Does any commutative complex unital Banach algebra have the $P(1, n)$ property for $n \geq 2$ ?

Problem 2. In particular does the disk algebra have the $P(1, n)$ property for $n \geq$ 2 ?

Problem 3. If some element $f$ of a commutative complex Banach algebra $\mathcal{A}$ does not belong to any regular maximal ideal of $\mathcal{A}$, does $\mathcal{A}$ have the $P(1, n)$ property for $n \geq 1$ ?

Problem 4. If the points of the maximal ideal space of such an algebra $\mathcal{A}$ are $G_{\delta}$, does $\mathcal{A}$ have the $P(k, n)$ property for all positive integers $k$ and $n$ ?

However, in 1989, Rao [1] showed that $P(2,3)$ does not hold for $C^{1}(B)$, the algebra of all continuously differentiable functions on $B$ where $B$ denotes the closed unit ball of $\mathbf{R}^{3}$. So the fourth problem of Jarosz is settled negatively. We give this example and modify it to give a negative answer to the third problem. However, the first two problems are still open.

Example 4.1 (11]). Let $\mathcal{A}=C^{1}(B)$. It is clear that the maximal ideal space of $\mathcal{A}$ is $B$. Let $P$ and $Q$ be arbitrary points of $B$ with $Q$ in the interior. Consider the three continuous linear functionals $\nu_{1}, \nu_{2}$ and $\nu_{3}$ defined by $\nu_{1}(f)=f(P)+f_{x}(Q)$, $\nu_{2}(f)=f(Q)$, and $\nu_{3}(f)=f_{x}(Q)+i f_{y}(Q)$ for all $f \in \mathcal{A}$, where $x, y, z$ denote the coordinates of a point in $\mathbf{R}^{3}$. We show that each element of the subspace

$$
M={ }^{\perp}\left[\nu_{1}, \nu_{2}, \nu_{3}\right]=\left\{f \in \mathcal{A}: \quad \nu_{1}(f)=\nu_{2}(f)=\nu_{3}(f)=0\right\}
$$

has at least two distinct zeros in $B$ while $M$ has only one common zero $Q$.

Let $f \in M$. If $f(P)=f(Q)=0$, we are done. If $f(P) \neq 0$, then also by the definition of $M, f_{x}(Q)$ and $f_{y}(Q)$ are not equal to zero. Write $f=g+i h$ where $g$ and $h$ are real valued functions in $\mathcal{A}$. Consequently $g_{x}(Q)=h_{y}(Q)$ and $g_{y}(Q)=-h_{x}(Q)$. Since $f_{x}(Q) \neq 0$, the previous two quantities are not equal to zero, i.e. $g_{x}(Q)=h_{y}(Q) \neq 0$. Therefore, by the implicit function theorem, there are two 2-dimensional $C^{1}$ manifolds passing through $Q$ such that $g=0$ on one of them and $h=0$ on the other. So on their intersection, which contains at least a $C^{1}$ curve passing through $Q, f$ is identically zero.

To show that Problem 3 of Jarosz has also a negative answer, we modify the above example.

Example 4.2. We use the same notations as in Example 4.1. Put

$$
\mathcal{A}_{0}=\{f \in \mathcal{A}: \quad f(Q)=0\} .
$$


It is not difficult to see that $\mathcal{A}_{0}$ is a non-unital commutative Banach algebra with the maximal ideal space $B^{\prime}=B \backslash\{Q\}$ and there are functions in $\mathcal{A}_{0}$ which are nonzero on $B^{\prime}$, i.e. do not belong to any regular maximal ideal of $\mathcal{A}_{0}$. Now put

$$
M_{0}={ }^{\perp}\left[\nu_{1}, \nu_{3}\right]=\left\{f \in \mathcal{A}_{0}: \nu_{1}(f)=\nu_{3}(f)=0\right\}
$$

in $\mathcal{A}_{0}$. Then $M_{0}$ is of codimension 2 in $\mathcal{A}_{0}$ and as in the above we see that every element of $M_{0}$ has at least one zero in $B^{\prime}$ but there is no common zero for $M_{0}$ in $B^{\prime}$. Therefore $P(1,2)$ does not hold for $\mathcal{A}_{0}$ and the answer to the third problem would also be negative.

Finally we treat the most important type of non-unital commutative Banach algebras $C_{0}(X)$, the algebra of all continuous complex-valued functions on a locally compact Hausdorff space $X$ vanishing at infinity.

If $X$ is not $\sigma$-compact, then every element of $C_{0}(X)$ has infinitely many zeros in $X$. So, it does not make any sense to speak about the $P(k, n)$ property in this case. But if $X$ is assumed to be $\sigma$-compact the $P(1, n)$ property follows for $C_{0}(X)$ from Theorem 2 of 8 for all $n \in \mathbf{N}$. If we further assume that all the points of $X$ are $G_{\delta}$, the $P(k, n)$ property follows for $C_{0}(X)$ from [7] for all $k, n \in \mathbf{N}$ by only considering the natural embedding of $C_{0}(X)$ into $C\left(X_{\infty}\right)$, where $X_{\infty}$ denotes the one point compactification of $X$.

Here we present a simple proof of $P(1,1)$ for $C_{0}(X)$ that is independent of $[8$ :

Theorem 4.3. Let $X$ be a locally compact Hausdorff space that is $\sigma$-compact. Then $C_{0}(X)$, the space of all complex valued functions on $X$ vanishing at infinity, satisfies $P(1,1)$.

Proof. First we show that there exists a positive function $f_{0}$ in $C_{0}(X)$. Write $X=$ $\bigcup_{n=1}^{\infty} K_{n}$, where $K_{n}$ is compact for each $n \geq 1$. By the Urysohn lemma we find a sequence $g_{n} \in C_{c}(X)$ such that $0 \leq g_{n} \leq 1$ and $g_{n}=1$ on $K_{n}$. Set $f_{0}=\sum_{i=1}^{\infty} \frac{g_{i}}{2^{i}}$. Then $f_{0} \in C_{0}(X)$ and $f_{0}>0$ on $X$.

Now, let $M$ be a closed subspace of $C_{0}(X)$ of codimension 1 such that every $f \in M$ has a zero in $X$. Then $M^{\perp}=\left[\mu_{0}\right]$ for some regular Borel measure $\mu_{0}$ on $X$. Hence

$$
M=\left\{f \in C_{0}(X): \int f d \mu_{0}=0\right\} .
$$

Note that $f_{0} \notin M$ because every element of $M$ vanishes at some point of $X$. Let $\alpha=\int f_{0} d \mu_{0}$. Clearly $\alpha \neq 0$. Replacing $\mu_{0}$ by $\alpha^{-1} \mu_{0}$ we obtain a new $\mu_{0} \perp M$ such that $\int f_{0} d \mu_{0}=1$. Now given $f \in C_{0}(X)$ we write $g=f-\left(\int f d \mu_{0}\right) f_{0}$. Then $\int g d \mu_{0}=\int f d \mu_{0}-\int f d \mu_{0}=0$. Therefore $g \in M$. Hence there exists $x_{0} \in X$ such that $g\left(x_{0}\right)=0$, i.e. $\int f d \mu_{0}=f\left(x_{0}\right) / f_{0}\left(x_{0}\right)$. Now if $f \geq 0$, then $\int f d \mu_{0} \geq 0$; hence $\mu_{0} \geq 0$.

We have shown that $\int f d \mu_{0} \in \operatorname{Im}\left(f / f_{0}\right)$ for every $f \in C_{0}(X)$. Replacing $f$ by $f f_{0}$ we get $\int f f_{0} d \mu_{0} \in \operatorname{Im}(f), f \in C_{0}(X)$. From this we conclude that $f_{0} d \mu_{0}=d \delta_{v}$, the point mass at some $v \in X\left[3\right.$, Lemma 2.5]. Write $E=X \backslash\{v\}$. Then $\int_{E} f_{0} d \mu_{0}=0$; hence $f_{0}=0$ a.e. $\mu_{0}$ on $E$. Because $f_{0}$ is strictly positive, we conclude that $\mu_{0}(E)=0$, that is, $\mu_{0}=c \delta_{v}$ for some constant $c>0$, so that,

$$
M=\left\{f \in C_{0}(X): f(v)=0\right\} .
$$

The proof is now complete. 


\section{REFERENCES}

[1] C. P. Chen, A generalization of the Gleason-Kahane theorem, Pacific J. Math. 107 (1983), 81-87. MR 85d:46070

[2] C. P. Chen and J. P. Cohen, Ideals of finite codimension in commutative Banach algebras, manuscript.

[3] F. Ershad and K. Seddighi, Multiplicative linear functionals in a commutative Banach algebra, Arch. Math. 65 (1995), 71-79. MR 96h:46079

[4] N. Farnum and R. Whitely, Functionals on real $C(S)$, Canad. J. Math. 30 (1978), 490-498. MR 57:13459

[5] R. V. Garimella and N. V. Rao, Closed subspaces of finite codimension in some function algebras, Proc. Amer. Math. Soc. 101 (1987), 657-661. MR 88m:46066

[6] A. M. Gleason, A characterization of maximal ideals, J. Analyse Math. 19 (1967), 171-172. MR 35:4732

[7] K. Jarosz, Finite codimensional ideals in function algebras, Trans. Amer. Math. Soc. 287 (1985), 725-733. MR 86c:46058

[8] - Finite codimensional ideals in Banach algebras, Proc. Amer. Math. Soc. 101 (1987), 313-316. MR 88h:46094

[9] — , Generalizations of the Gleason-Kahane-Zelazko theorem, Rocky Mountain J. Math. 21 (1991), 915-921. MR 92m:46003

[10] J. P. Kahane and W. Zelazko, A characterization of maximal ideals in commutative Banach algebras, Studia Math. 29 (1968), 339-343. MR 37:1998

[11] N. V. Rao, Closed ideals of finite codimension in regular selfadjoint Banach algebras, $J$. Funct. Anal. 82 (1989), 237-258. MR 90g:46077

[12] C. R. Warner and R. Whitely, A characterization of regular maximal ideals, Pacific J. Math. 30 (1969), 277-281. MR 54:3420

[13] Ideals of finite codimension in $C[0,1]$ and $L^{1}(\mathbf{R})$, Proc. Amer. Math. Soc. 76 (1979), 263-267. MR 81b:46070

Department of Mathematics, Shiraz University, Shiraz 71454, Iran

Department of Mathematics, Shiraz University, Shiraz 71454, Iran

E-mail address: shir@sun01.susc.ac.ir 